\title{
INFORMATIONEN
}

Die Informationen in den folgenden Rubriken beruhen auf

Meldungen der mathematischen Institute/Fachbereiche.

NEUE MITGLIEDER

\section{NUR IN DER DRUCKFASSUNG}

\section{TODESFÄLLE}

Herr Prof. em. Dr. Karl Kießwetter (Ahrensburg) ist am 21. 9. 2019 verstorben.

Herr Prof. em. Dr. Harald Holmann (Fribourg) ist am 12. 3. 2020 verstorben.

Herr Prof. em. Dr. Winfried Scharlau (Münster) ist am 26. 11. 2020 verstorben.
Frau Prof. em. Dr. Elke Dagmar Heinrich (Lübeck) ist am 21. 1. 2021 verstorben.

\section{HABILITATIONEN}

Tcheutia, Daniel Duviol (Kassel): Algorithmic methods for mixed recurrence equations, zeros of classical orthogonal polynomials and classical orthogonal polynomial solutions of three-term recurrence equations. Koepf, Marcellán, Foupouagnigni, 17.07.2019

Werner, Frank (Göttingen): Statistical inverse problems - Regularization and inference. Clason, Reiss, Vanhems, 11.02.2020

\section{PROMOTIONEN}

Universität Bayreuth

Ortiz López, Julián: Constrained optimization on manifolds. 26.11.2020.

Stöcklein, Matthias: Optimal control of static contact in finite strain elasticity. 15.12.2020.

Universität Bremen

Intemann, Timm: Dreistufige Verfahren zur Modellierung von Ernährungsdaten. Pigeot-Kübler, Didelez 20.07.20.

Donau, Ralf: Combinatorial topology of quotients of posets. Feichtner-Kozlov, Gamst 04.08.20.

Brandt, Carsten : Recurrence quantification compared to Fourier analysis for ultrasonic non-destructive testing of carbon fibre reinforced polymers. Maaß, Marwan 30.06.20.

Langeheine, Malte: Investigating participation in population - based cohort studies using paradata. Ahrens, Didelez 23.06.2020.

\section{Technische Universität Chemnitz}

Rebs, Christian: Asymptotic bounds and values for the norm of the Laplace operator and other partial differential operators on spaces of polynomials. Prof. Böttcher, Prof. Kunis, Prof. Roch 23.06.2020.

Hofmann, Christopher: Contributions to regularization theory and practice of certain nonlinear inverse problems. Hofmann, Kindermann, Plato 11.12.2020. 
DMV-Ansprechpartner/innen vor Ort

- RWTH Aachen: Gabriele Nebe

- Universiteit van Amsterdam: Benedikt Löwe

- U Augsburg: Bernhard Hanke

- U Bamberg: Anna-Susanne Steinweg

- U Bayreuth: Michael Stoll

- FU Berlin: Christian Haase

- TU Berlin: Martin Skutella

- HU Berlin: Jürg Kramer

- WIAS Berlin: Wolfgang König

- U Bielefeld: Michael Röckner

- FH Bielefeld: Claudia Cottin

- Hochschule Bochum: Thomas Skill

- Ruhr-U Bochum: Peter Eichelsbacher

- U Bonn: Daniel Huybrechts

- TU Braunschweig: Volker Bach

- Jacobs U Bremen: Marcel Oliver

- U Bremen: Anke Pohl

- TU Chemnitz: Christoph Helmberg

- BTU Cottbus: Friedrich Sauvigny

- TU Darmstadt: Stefan Ulbrich

- TU Dortmund: Ben Schweizer

- TU Dresden: Andreas Thom

- U Düsseldorf: Kai Köhler

- U Erlangen-Nürnberg: Günter Leugering

- U Duisburg-Essen, Campus Essen: Rüdiger Schultz

- U Flensburg: Hinrich Lorenzen

- U Frankfurt: Thorsten Theobald

- TU Bergakademie Freiberg: Michael Eiermann

- U Freiburg: Sebastian Goette

- U Gießen: Thomas Bartsch

- U Göttingen: Thomas Schick

- U Greifswald: Michael Schürmann

- FernUni Hagen: Winfried Hochstättler

- U Halle-Wittenberg: Rebecca Waldecker

- TU Hamburg-Harburg: Wolfgang Mackens

- Leibniz U Hannover: Christine Bessenrodt

- U Heidelberg: Gebhard Böckle

- U Hildesheim: Jürgen W. Sander
- U Hohenheim: Georg Zimmermann

- TU Ilmenau: Carsten Trunk

- U Jena: Tobias Oertel-Jäger

- KIT Karlsruhe: Michael Plum

- U Kassel: Wolfram Koepf

- U zu Köln: Peter Littelmann

- U Konstanz: Oliver Schnürer

- Hochschule Landshut: Konstantin Ziegler

- U Leipzig: Hans-Bert Rademacher

- Uzu Lübeck: Jürgen Prestin

- Leuphana U Lüneburg: Silke Ruwisch

- U Magdeburg: Volker Kaibel

- U Mainz: Martin Hanke-Bourgeois

- U Mannheim: Leif Döring

- U Marburg: Volkmar Welker

- U München: Helmut Schwichtenberg

- TU München: Peter Gritzmann

- U der Bundeswehr München: Cornelius Greither

- U Münster: Michael Joachim

- HS Neubrandenburg: Gerd Teschke

- U Oldenburg: Daniel Grieser

- U Osnabrück: Holger Brenner

- U Paderborn: Margit Rösler

- U Passau: Brigitte Forster-Heinlein

- U Potsdam: Christian Bär

- U Regensburg: Guido Kings

- U Rostock: Roger Labahn

- U des Saarlandes: Jörg Eschmeier

- U Siegen: Thorsten Raasch

- HS für Technik (HFT) Stuttgart: Peter Hauber

- U Stuttgart: Timo Weidl

- U Trier: Jochen Wengenroth

- U Tübingen: Carla Cederbaum

- Hochschule Ulm: Günter Gramlich

- Bauhaus U Weimar: Klaus Gürlebeck

- Bergische U Wuppertal: Jens Hornbostel

- U Würzburg: Stefan Waldmann

\section{Goethe-Universität Frankfurt}

Jablonski, Simone: Wie potenziell mathematisch begabte Kinder argumentieren. Ludwig, Brunner 11.12.2020.

Bieri, Maximilian: On fibrations approaching the Arakelov equality. Möller, Zuo 13.11.2020.

Lüdtke, Martin: Stix, Werner. The $p$-adic section conjecture for localisations of curves 14.12.2020.

Knörr, Jonas: Smooth valuations on convex functions. Bernig, Sanyal 11.12.2020.
Albert-Ludwigs-Universität Freiburg

Hou, Yuhang: Elliptic genera of ADE type singularities. Wendland, Taormina 18.12.2020.

\section{Georg-August-Universität Göttingen}

Mukherjee, Devarshi: Topological Invariants for NonArchimedian Bornological Algebras. Schick, Kremnitzer, Clausen 24.09.2020.

Ewert, Eske Ellen: Index Theory and Groupoids for Filtered Manifolds. Schick, Schrohe, Moller 26.10.2020. 
Suchla, Engelbert: $L^{2}$-invariants for self-similar $C W$ complexes. Schick, Bahns, Elek 07.10.2020.

Dehling, Malte: Symmetric homotopy theorie for operads and weak Lie 3-algebras. Zhu, Vallette 16.11.2020.

Hansen, Nils Bahne: Structure theory of the PohlmeyerRehren Lie algebra and adaptations of the Hall algorithm to non-free graded Lie algebras. Bahns, Rehren 18.11.2020.

\section{Universität Hamburg}

Hida, Takanori: Topics in iteration theory. Zerr, Geschke 23.12.2020.

Vogrin, Martin: Algebraic structures on moduli spaces of mirror geometries. Alim, Movasati 20.01.2021.

Thung, Daniel Konstantin: Symmetries of deformed c-map metrics and the HK/QK correspondence. Cortes, Heber, Haydys 01.12.2020.

\section{Ruprecht-Karls-Universität Heidelberg}

Gräf, Peter Mathias: Boundary distributions for $\mathrm{SL}_{3}$ over a local field and symmetric power coefficients. 18.12.2020.

Schluttenhofer, Sandra Simone: Adaptive minimax testing for inverse problems. 14.12.2020.

Jost, Jan Niklas: Mirror symmetry for Del Pezzo surfaces. 26.01.2021.

\section{Technische Universität Kaiserslautern}

Hambardzumyan, Hayk: Dynamic hybrid pension products with innovative embedded guarantee structures and optimal portfolio strategies. Korn, Weber 20.07.2020.

Sieber, Alexander: On the complexity and approximability of optimization problems with minimum quantity constraints. Krumke, Stiller 18.08.2020.

Arioli, Clarissa: Isogeometric analysis for scaled boundary parametrizations. Simeon, Möller 25.08.2020.

Gottschalk, Simon: A differential equation based framework for deep reinforcement learning. Simeon, Gerdts 27.08.2020.

Kusch, Lisa: Robustness measures and optimization strategies for multi-objective robust design. Gauger, Walther o8.09.2020.

Shamanskiy, Alexander: Mesh deformation in the context of isogeometric analysis. Simeon, Jüttler 11.09.2020.

Corbin, Gregor: Numerical methods for multi-scale cell migration models - Applied to glioma invasion. Klar, Frank 28.09.2020.

Epure, Raul-Paul: Explicit and effective Mather-Yau correspondence in view of analytic gradings. Schulze, Hauser 13.11.2020.

Wang, Jingnan: Reflected anticipated backward stochastic differential equitations with default risk, numerical algorithms and applications. Korn, Neuenkirch 20.11.2020.

Koch, Lisa Ingrid: Rational interpolation in the loewner framework, optimal PMOR and aspects of IRKA. Damm, Faßbender 24.01.2020.
Nonnenmacher, Andreas: Operator semigroups and infinite dimensional analysis applied to problems from mathematical physics. Grothaus, Albeverio 02.10.2020.

Kocoglu, Damla: Analysis of systems of hyperbolic partial differential equations coupled to switched differential algebraic equations. Trenn, Hante 11.12.2020.

Karlsruher Institut für Technologie

Kusch, Jonas: Realizability-preserving discretization strategies for hyperbolic and kinetic equations with uncertainty. Frank, Wieners, McClarren 06.05.2020.

Maier, Bernhard: Error analysis for space and time discretizations of quasilinear wave-type equations. Hochbruck, Schnaubelt, Makridakis 27.05.2020.

Herold, Felix: Random mosaics in hyperbolic space. Hug, Last 17.06.2020.

Caspart, Sven: Singularities of translation manifolds. Herrlich, Weitze-Schmithüsen 01.07.2020.

Waßermann, Benjamin: The $L^{2}$-Cheeger-Müller theorem for representations of hyperbolic lattices. Sauer, Lamm, Pozzetti 30.07.2020.

Krämer, Julian: A hybrid weakly conforming finite element method for applications in solid mechanics. Wieners, Dörfler 23.09.2020.

Klemens, Fabian: Combining computational fluid dynamics and magnetic resonance imaging data using lattice Boltzmann based topology optimisation. Thäter, Dörfler, Krause 30.09.2020.

Glauner, Alexander: Robust and risk-sensitive Markov decision processes with applications to dynamic optimal reinsurance. Bäuerle, Jaśkiewicz 11.11.2020.

Freese, Jan Philip: Numerical homogenization of timedependent Maxwell's equations with dispersion effects. Wieners, Gallistl 25.11.2020.

Schmid, Tobias: Local wellposedness and global regularity results for biharmonic wave maps. Lamm, Schörkhuber, Krieger 16.12.2020.

Zerulla, Konstantin: ADI schemes for the time integration of Maxwell equations. Schnaubelt, Hochbruck 16.12.2020.

\section{Universität Kassel}

Hohmann, Raphael Mike: Continuous adjoint-based shape optimization for particle transport problems in fluid. Meister, Pinnau 19.02.2020.

Hock, Natalie: Förderung der Fehler-Ursachen-

Diagnosekompetenz von Mathematiklehramtsstudierenden.

Borromeo Ferri, Besser, Blum 11.05.2020.

Teguia Tabuguia, Bertrand: Power series representations of hypergeometric type and non-holonomic functions in computer algebra. Koepf, Seiler 27.05.2020.

Nkotto Nkung Assong, Sedric: Explicit description of isogeny and isomorphism classes of drinfeld modules of higher rank over finite fields. Rück, Petersen 01.07.2020. 
Straub, Veronika: Effiziente Zeitintegration vom gebietsbasierten implizit-expliziten Typ auf Basis exponentieller Integratoren zur Simulation kompressibler instationärer Strömungen. Meister, Birken 09.10.2020.

\section{Otto-von-Guericke-Universität Magdeburg}

Marius, Schmidt: Optimale Versuchsplanung für Zähldaten mit zufälligen Blockeffekten. Schwabe, Müller 27.01.2020.

Mlinaric, Petar: Structure-preserving model order reduction for network systems. Benner, Trentelman 23.01.2020.

Röttger, Frank: Geometry of optimal design and limit theorems. Schwabe, Wynn, Kahle 10.06.2020.

Borger, Christopher: Mixed lattice polytope theory with a view towards sparse polynomial systems. Nill, Averkov, Leal 11.06.2020.

Ananiadi, Lamprini: Symmetry in toric geometry. Kahle, Römer 31.08.2020.

Mierswa, Alina: Error extimates for a finite difference approximation of mean curvature flow for surfaces of torus type. Deckelnick, Styles 13.10.2020.

Jost, Felix: Model-based optimal treatment schedules for acute leukemia. Sager, Lebiedz 26.10.2020.

Munir, Taj: Analysis of coupling interface problems for bi-domain diffusion equations. Warnecke, Chamakuri

30.11.2020.

Gerike, Daniel: Determining the Cycle Structure of Permutation Polynomials of Shape $X^{t}+\gamma \operatorname{Tr}\left(X^{k}\right)$. Pott, Kyureghyan, Topuzoglu 22.06.2020.

Johannes Gutenberg-Universität Mainz

Felten, Simon: Log toroidal families. Ruddat, van Straten 14.01.2021.

\section{Universität Passau}

Hatzesberger, Simon: Strongly asymptotically optimal methods for the pathwise global approximation of stochastic differential equations with coefficients of super-linear growth. Müller-Gronbach, Sabanis 21.2.2020.

Horácek, Jan: Algebraic and logic solving methods for cryptanalysis. Kreuzer, Biere 12.02.2020.

\section{Universität Trier}

Hoffmann, Daniel: Stochastic particle systems and optimization - Branching processes, mean field games and impulse control. Seifried, Schulz, Christensen 28.10.2020.

\section{Universität zu Köln}

Uran, Matthias: High-performance computing two-scale finite element simulations of a contact problem using computational homogenization - Virtual forming limit curves for dual-phase steels. Klawonn, Rheinbach 18.05.2020.

Hochmuth, Christian : Parallel overlapping Schwarz preconditioners for incompressible fluid flow and fluid-structure interaction problems. Klawonn, Rheinbach 18.05.2020.
Prévost, Alexis: Percolation for the Gaussian free field and random interlacements via the cable system. Drewitz, Mörters 18.05.2020.

Licón-Saláiz, José Luis: Topological aspects of turbulence in the planetary boundary layer. Kunoth, Neggers 04.02.2020.

Jan Martin, Krämer: Regularity and symmetry results for ground state solutions of quasilinear eliptic equations. Kawohl, Sweers 24.06.2020.

Christian, Steinert: Fano varieties and Fano polytopes. Littelmann, Fourier 08.09.2020.

Ciolan, Emil-Alexandru: Asymptotics, equidistribution and inequalities for partion functions. Bringmann, Zwegers 23.06.2020.

Schnieders, Inka: Positivity and regularity of solutions to higher order Dirichlet problems on smooth domains. Sweers, Horstmann 12.01.2021.

\section{Julius-Maximilians-Universität Würzburg}

Roos, Anna-Katharina: Mathematisches Begriffsverständnis im Übergang Schule-Universität am Beispiel des Extrempunktbegriffs. Weigand, Ulm 09.03.2020.

Kann, Lennart: Statistical failure prediction with an account for prior information. Göb, Weiß 02.06.2020.

Seifert, Bastian: Multivariate Chebyshev polynomials and FFT-like algorithms. Hüper, Munthe-Kaas, Püschel 10.07.2020.

Fuller, Timo: Contributions to the multivariate max-domain of attraction. Falk, Stupfler 13.07.2020.

Rehberg, Martin: Weighted uniform distribution related to primes and the Selberg Class. Steuding, Bruchlos, Sander 31.07.2020.

Manderfeld, Katharina: Was ist Mathematikdidaktik? - Explorative Studien zu Vorstellungen von Bachelorstudierenden im Lehramt Mathematik. Siller, Rolka 11.09.2020.

Lung, Jennifer: Schulcurriculares Fachwissen von Mathematiklehramtsstudierenden - Struktur, Entwicklung und Einfluss auf den Studienerfolg. Siller, Drüke-Noe 14.09.2020.

Klock, Heiner: Adaptive Interventionskompetenz in mathematischen Modellierungsprozessen. Konzeptualisierung, Operationalisierung und Förderung. Siller, Greefrath 21.09.2020.

Suttner, Raik: Output optimization by Lie bracket approximations. Dashkovskiy, Zuyev, Ebenbauer 25.09.2020.

Wisheckel, Florian: Some applications of D-norms to probability and statistics. Falk, Guillou 02.12.020.

Karl, Veronika: Augmented Lagrangian methods for state constrained optimal control problems. Wachsmuth, Neitzel 21.10.2020.

Lauerbach, Laura: Stochastic homogenization in the passage from discrete to continuous systems - fracture in composite materials. Schlömerkemper, Kružík 26.10.2020. 
Börgens, Eike: ADMM-type methods for optimization and generalized Nash equilibrium problems in Hilbert spaces.

Kanzow, Boţ 14.12.2020.
Berberich, Jonas: Fluids in gravitational fields - Wellbalanced modifications for astrophysical finite-volume codes. Klingenberg, Chandrashekar 11.01.2021.

\section{DEUTSCHE MATHEMATIKER-VEREINIGUNG}

- VORSTAND UND PRÄSIDIUM Präsident Prof. Dr. Friedrich Götze, Fakultät für Mathematik, Universität Bielefeld, Universitätsstraße 25, 33615 Bielefeld, Tel. +49.521 106-4774 goetze@math. uni-bielefeld.de Schatzmeister Prof. Dr. Etienne Emmrich, Institut für Mathematik, MA 5-3, Technische Universität Berlin, Straße des 17. Juni 136, 10623 Berlin, Tel. +49.30314 25745, emmrich@math.tu-berlin.de Schriftführer Prof. Dr. Daniel Grieser, Universität Oldenburg, Institut für Mathematik, Carl-vonOssietzky-Straße 9-11, 26129 Oldenburg, Tel. +49 · 441 $\cdot 7983230$ daniel.grieser@uni-oldenburg.de Herausgeber der Mitteilungen Prof. Dr. Sebastian Stiller (verantwortlich), Technische Universität Carolo-Wilhelmina zu Braunschweig, Universitätsplatz 2, 38106 Braunschweig, sebastian.stiller@tu-bs.de Weitere Präsidiumsmitglieder . Prof. Dr. Ilka Agricola, Marburg (Nachwuchsförderung) - Prof. Dr. Bernhard Hanke, Augsburg (Gauß-Vorlesung) . Prof. Dr. Moritz Kaßmann, Bielefeld (Industrie- und Unternehmenskontakte) - Prof. Dr. Wolfram Koepf, Kassel (Vorsitzender der Kommission Übergang Schule-Hochschule) . Prof. Dr. Frank Loose, Tübingen (Kontakte in Schule und Hochschule) . Prof. Dr. Guido Schneider, Stuttgart (Herausgeber des Jahresberichtes der DMV) - Matthias Lippert, Remscheid (Fragen von Schule und Lehrkräftebildung) . Prof. Dr. Günter M. Ziegler, FU Berlin (Leiter Medienund Netzwerkbüro der DMV) - Mitgliedsbeitrag 2020 (inkl. Bezug der Mitteilungen und einer gewählten Zeitschrift, Ausnahme: Studierende und Schüler beziehen nur die Mitteilungen) - regulär EUR 105,00 - bis zur Vollendung des 30. Lebensjahres EUR 50,00 - ermäßigt für Ehepaare und eingetragene Lebenspartnerschaften EUR 150,00 - ermäßigt für Studierende (Bachelor/Master/Diplom) und Schülerinnen und Schüler EUR 20,00 - Sonderbeitrag auf Antrag (z. B. bei Arbeitslosigkeit) EUR 30,00 . ermäßigt für Mitglieder der DPG/GI/GOR/GDM/MNU oder MUED EUR 90,oo EUR . ermäßigt für Reziprozitätsmitglieder (im Ausland wohnend und Vollmitglied einer Mathematischen Gesellschaft, mit der die DMV ein Reziprozitätsabkommen hat) EUR 70,00 . ermäßigt für Senioren EUR 70,00 — Zeitschriften (Jahresabo 2020 jeweils EUR 28,oo), eine der folgenden Zeitschriften ist im Mitgliedsbeitrag enthalten: - Jahresbericht der DMV (Springer Verlag Heidelberg, 4 Hefte jährlich) . Mathematische Semesterberichte (Springer Verlag Heidelberg, 2 Hefte jährlich) - Journal für MathematikDidaktik (Springer Verlag Heidelberg, 2 Hefte jährlich) — DMVServer www.mathematik.de - DOCUMENTA MATHEMATICA www.mathematik.uni-bielefeld.de/documenta/ - Medienbüro der DMV Thomas Vogt, FU Berlin (mathematik.de) . Netzwerkbüro der DMV Beate Klompmaker, FU Berlin (mathematik.de) - Geschäftsstelle der DMV Geschäftsführerin Andrea KirsteinGaekel (mathematik.de) - Bankverbindung Volksbank Freiburg 6955002 (BLZ 680 900 oo), IBAN: DE66 6809000000069550 o2, BIC: GENODE61FR1

Die Deutsche Mathematiker-Vereinigung e. V. ist durch den Freistellungsbescheid für 2016 bis 2018 des Finanzamtes für Körperschaften Berlin I (Steuer-Nr. 27/640/51051) vom 27.1.2020 wegen „Förderung von Wissenschaft und Forschung" als wissenschaftlichen Zwecken dienend und zu den in $\S_{5}$ Absatz 1 Nr. 9 KStG bezeichneten Körperschaften gehörig anerkannt worden. Vereinseintrag: VR 380040 beim Amtsgericht Stuttgart. UmsatzsteuerIdentifikationsnummer: DE 165534138.
IMPRESSUM - Verleger Walter de Gruyter GmbH, Berlin/Boston, www.degruyter.com - Herausgeber Prof. Dr. Sebastian Stiller (verantwortlich), Institut für Mathematische Optimierung, Technische Universität Carolo-Wilhelmina zu Braunschweig, Universitätsplatz 2, 38106 Braunschweig, sebastian. stiller@tu-bs.de — Prof. Dr. Michael Joswig, Fakultät II - Mathematik und Naturwissenschaften, Institut für Mathematik, MA 6-2, TU Berlin, Straße des 17. Juni 136, 10623 Berlin, joswig@math.tu-berlin.de • Prof. Dr. Brigitte Lutz-Westphal, Institut für Mathematik, Freie Universität Berlin, Arnimallee 3, 14195 Berlin, brigitte.lutz-westphal@math.fu-berlin.de - Prof. Günter M. Ziegler, Institut für Mathematik, FU Berlin, Arnimallee 2, 14195 Berlin, ziegler@math.fu-berlin.de - Redaktion Christoph Eyrich, Silke Thiel, Thomas Vogt, mdmv@ math.tu-berlin.de - Adresse der Redaktion Mitteilungen der DMV, Institut für Mathematik, FU Berlin, Arnimallee 2, 14195 Berlin, Tel. +49.30.8387566o mdmv@math.tu-berlin.de - Grafische Gestaltung und Satz Christoph Eyrich, Berlin - Druck Grafisches Centrum Cuno, Calbe n Erscheinungsweise vierteljährlich. Der Bezugspreis ist im Mitgliedsbeitrag der DMV enthalten. Manuskripte senden Sie bitte an den Herausgeber.

Bitte senden Sie Adressenänderungen und alle die Mitgliedschaft betreffenden Zuschriften an die Geschäftsstelle der DMV, c/o WIAS, Mohrenstraße 39, 10117 Berlin, Tel. +49.30.20372-306, Fax +49.30.20372-307, dmv@wias-berlin.de I Namentlich gekennzeichnete Beiträge geben nicht unbedingt die Meinung der Redaktion wieder. 